\title{
溶液中类锗烯 $\mathrm{H}_{2} \mathrm{GeClMgCl}$ 的结构与异构化反应
}

\author{
李文佐 ${ }^{1, *}$ 程建波 ${ }^{1}$ 李庆忠 ${ }^{1}$ 宫宝安 ${ }^{1}$ 孙家钟 ${ }^{1,2}$ \\ $\left({ }^{1}\right.$ 烟台大学化学生物理工学院, 山东 烟台 264005; 2 吉林大学理论化学计算国家重点实验室, 长春 130012)
}

摘要：用密度泛函理论(DFT)和 QCISD(quadratic configuration interaction with single and double excitations)方 法研究了类锗烯 $\mathrm{H}_{2} \mathrm{GeClMgCl}$ 在气相和五种溶剂中的构型与异构化反应. 结果表明, 类锗烯 $\mathrm{H}_{2} \mathrm{GeClMgCl}$ 有三 种平衡构型. 其中 $p$-配合物型构型能量最低, 是其存在的主要构型. 讨论了溶剂效应对结构、能量与异构化反 应的影响. 计算模拟了最稳定构型的红外光谱.

关键词：类锗烯 $\mathrm{H}_{2} \mathrm{GeClMgCl} ; \mathrm{B} 3 \mathrm{LYP} ; \mathrm{QCISD} ;$ 异构化; 溶剂效应

中图分类号: 0641.12

\section{Structures and Isomerization Reactions of the Germylenoid $\mathrm{H}_{2} \mathrm{GeClMgCl}$ in Solution}

\author{
LI Wen-Zuo ${ }^{1, *} \quad$ CHENG Jian-Bo ${ }^{1} \quad$ LI Qing-Zhong ${ }^{1} \quad$ GONG Bao-An ${ }^{1} \quad$ SUN Jia-Zhong ${ }^{1,2}$ \\ ('Science and Engineering College of Chemistry and Biology, Yantai University, Yantai 264005, Shandong Province, P. R. China; \\ ${ }^{2}$ State Key Laboratory of Theoretical and Computational Chemistry, Jilin University, Changchun 130021, P. R. China)
}

\begin{abstract}
The germylenoid $\mathrm{H}_{2} \mathrm{GeClMgCl}$ was studied by using density functional theory (DFT) and quadratic configuration interaction with single and double excitations (QCISD) method for the gas phase and in five solvents. Geometry optimization calculations indicated that $\mathrm{H}_{2} \mathrm{GeClMgCl}$ had three equilibrium configurations and the $p$ complex structure was the lowest in energy and was the most stable structure. The solvent effect on the geometries, energies, and isomerization reactions were discussed. The infrared spectrum was simulated for the most stable structure.
\end{abstract}

Key Words: Germylenoid $\mathrm{H}_{2} \mathrm{GeClMgCl}$; B3LYP; QCISD; Isomerization; Solvent effect

类锗烯 (germylenoid) 是类卡宾 (carbenoid)与类 硅烯 (silylenoid) 的类似物, 可以用通式 $\mathrm{R}_{1} \mathrm{R}_{2} \mathrm{GeMX}$ ( $X$ 通常为卤素, $M$ 通常为碱金属)来表示. 1991 年, Gaspar 等人 ${ }^{[1]}$ 提出在四氢呋喃(THF)溶液中, 二氯二 甲基锗烷与取代的 1,3-丁二烯反应时, 可能存在某 种类锗烯作为反应的中间体. 后来的一系列实验 ${ }^{[2-4]}$ 提出了类似的论断. 由于许多锗类化合物具有生物 活性 ${ }^{[5-8]}$, 如何设计合成新的锗类化合物越来越引起 人们的兴趣. 而类锗烯作为一类重要的有机锗反应
中间体, 在新的锗类化合物合成中有着潜在的应用 价值, 因而日益受到人们的重视. 但至今实验上仍 未成功合成稳定的类锗烯. 对类锗烯进行系统的理 论研究对正确理解类错烯的结构、性质和反应性很 有必要. 自从 1999 年 Qiu 等人 ${ }^{[9]}$ 首次对最简单的类 锗烯 $\mathrm{H}_{2} \mathrm{GeLiF}$ 进行了研究, 现已较系统的研究了类 锗烯 $\mathrm{H}_{2} \mathrm{GeMX}$ 和 $\mathrm{CH}_{2} \mathrm{GeMX}(\mathrm{M}=\mathrm{Li}, \mathrm{Na} ; \mathrm{X}=\mathrm{F}, \mathrm{Cl}$ ) 各 种可能的异构体构型、稳定性以及相互转化的过渡 态 ${ }^{[10-16]}$. 上述研究主要集中在含碱金属原子的类锗

Received: July 21, 2008; Revised: October 10, 2008; Published on Web: November 17, 2008.

*Corresponding author. Email: liwenzuo2004@126.com; Tel: +86535-6902063.

国家自然科学基金(20473029)和烟台大学博士科研基金(HY05B30, HY05B36)资助项目

C. Editorial office of Acta Physico-Chimica Sinica 
烯上, 对含其它金属原子的类锗烯的研究尚不多 见 ${ }^{[17]}$. 为进一步扩展类锗烯的研究内容, 有必要对含 其它金属原子的类锗烯进行研究. 另一方面, 由于有 关类锗烯的实验研究均是在溶液中进行的, 对类锗烯 的理论研究也应考虑溶剂效应的影响. 尽管 $\mathrm{Ma}$ 等 人 ${ }^{[13]}$ 曾经研究过若干溶剂对简单类锗烯 $\mathrm{H}_{2} \mathrm{GeLiF}$ 结 构的影响, 但对溶液中类锗烯的结构、性质及反应 性的研究仍不系统. 基于上述考虑, 我们对含 $\mathrm{Mg}$ 原子的类锗烯 $\mathrm{H}_{2} \mathrm{GeClMgCl}$ 在气相和五种溶剂中 的结构与异构化反应进行了理论计算, 以进一步丰 富类锗烯的研究内容.

\section{1 计算方法}

对气相和五种溶液中的类锗烯 $\mathrm{H}_{2} \mathrm{GeClMgCl}$ 的平衡构型及构型间异构化反应的过渡态均采用 DFT(density functional theory) ${ }^{[18,19]}$ 和 B3LYP 方法 ${ }^{[20,21]}$ 进行全参数优化, 并进行振动频率及 IRC $^{[22,23]}$ (intrinsic reaction coordinate) 计算以确证过渡态, 以 上计算选择 6-311+ $\mathrm{G}(d, p)$ 基组 ${ }^{[24]}$. 为进一步考虑相 关能, 在优化构型上对各个物种进行了 QCISD (quadratic configuration interaction with single and

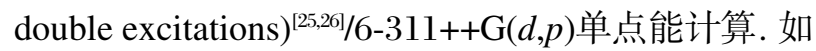
无特殊说明, 文中能量均指 QCISD/6-311++G $(d, p)$ $/ / \mathrm{B} 3 \mathrm{LYP} / 6-311+\mathrm{G}(d, p)$ 水平上计算所得的能量, 并在 $\mathrm{B} 3 \mathrm{LYP} / 6-311+\mathrm{G}(d, p)$ 水平上进行零点能校正 ${ }^{[16,27]}$. 采 用的溶剂化模型为Onsager 模型 ${ }^{[22,29]}$. 五种溶剂分别 为苯 (benzene), 乙醚 (diethylether), 四氢呋喃(THF), 丙酮(aacetone)和二甲基亚砜(DMSO), 介电常数分别 为 $2.247 、 4.335 、 7.58 、 20.7$ 和 46.7. 计算采用 Gaussian 03 程序 ${ }^{[30]}$.

\section{2 结果和讨论}

\section{$2.1 \mathrm{H}_{2} \mathrm{GeClMgCl}$ 的平衡构型}

B3LYP/6-311+G $(d, p)$ 构型优化与频率计算结 果表明, 在气相和五种溶液中 $\mathrm{H}_{2} \mathrm{GeClMgCl}$ 均有三 种平衡构型(图 1 中 1-3). 各平衡构型, 过渡态构型 见图 1. 各构型的结构参数列于表 1 .

图 1 中的构型 $\mathbf{1}$, 是缺电子带正电荷的 $\mathrm{Mg}$ 端 与 $\mathrm{Ge}$ 原子的 $\sigma$ 轨道结合, 富电子带负电荷的 $\mathrm{Cl}$ 端与 $\mathrm{Ge}$ 原子的空 $p$ 轨道结合形成的三元环状结 构. 该构型属于 $C_{s}$ 点群. 自然键轨道分析计算表明 在形成构型 1 时, $\mathrm{Ge}$ 上的电荷向 $\mathrm{Mg}$ 迁移, 而 $\mathrm{Mg}$ 上的电荷向 $\mathrm{Cll}$ 迁移, 这应该是构型 $\mathbf{1}$ 能够稳定存
在的主要原因. 气相下构型 $\mathbf{1}$ 的能量比能量最低构 型 2 的能量高 $24.10 \mathrm{~kJ} \cdot \mathrm{mol}^{-1}$.

构型 2 也属于 $C_{s}$ 点群, 可看作带负电的 $\mathrm{Cl}$ 端 进攻 $\mathrm{Ge}$ 的 $p$ 空轨道产生的构型, 在该构型中, $\mathrm{Cl}$ 上的电子向 $\mathrm{Ge}$ 的 $p$ 空轨道迁移, 可称之为 $p$-配合 物型构型. 构型 2 中带正电的 $\mathrm{Mg}$ 原子与带负电的 两个 $\mathrm{H}$ 原子间也有相互作用(见图 1), 这也是构型 2 能够稳定存在的原因之一. 构型 2 是能量最低的 构型.

构型 3 是变形的“四面体”构型, 也属于 $C_{s}$ 点 群, 可看作由于 $\mathrm{H}_{2} \mathrm{Ge}$ 的插人导致 $\mathrm{Mg}-\mathrm{Cll}$ 键断裂 产生的构型. 构型 3 是能量最高的构型, 气相下其 能量比能量最低构型 2 的能量高 $47.03 \mathrm{~kJ} \cdot \mathrm{mol}^{-1}$.

综上所述, 类锗烯 $\mathrm{H}_{2} \mathrm{GeClMgCl}$ 共有三种平衡 构型, 三种平衡构型均可视为 $\mathrm{H}_{2} \mathrm{Ge}$ : 与 $\mathrm{MgCl}_{2}$ 的加成 物. 从各平衡构型能量上分析 (见表 2 ), $\mathrm{H}_{2} \mathrm{GeClMgCl}$ 三种平衡构型的热力学稳定次序为 $2>1>3$.

\section{2 构型间的异构化反应及动力学稳定性}

构型 $4 、 5$ 和 6 是 $\mathrm{H}_{2} \mathrm{GeClMgCl}$ 势能面上的三 个过渡态, 由它们的反应矢量(能量二阶导数的唯一 负本征值对应的本征矢)及 IRC 计算结果分析可知, 它们分别为构型 1 与 $2 、 1$ 与 3 及 2 与 3 之间相互 异构化的过渡态. 振动分析计算表明, 构型 4.5 和 6 均存在唯一的虚频. 在 B3LYP/6-311+G $(d, p)$ 水平 上, 气相下 $4 、 5$ 和 6 的虚频分别为 $932.8 \mathrm{i} 、 71.2 \mathrm{i}$ 和 $910.3 \mathrm{i} \mathrm{cm}^{-1}$, 从而确证为真正的过渡态. 为了确证过 渡态与稳定几何构型的连接, 以得到的过渡态为起 始点, 沿着反应途径分别向前和向后进行了 IRC 计算, 结果表明过渡态结构与稳定几何构型的连接 是正确的.

一般说来, 各平衡构型的稳定性取决于它们自 身能量的高低及相互异构化的活化能. 由表 2 可以

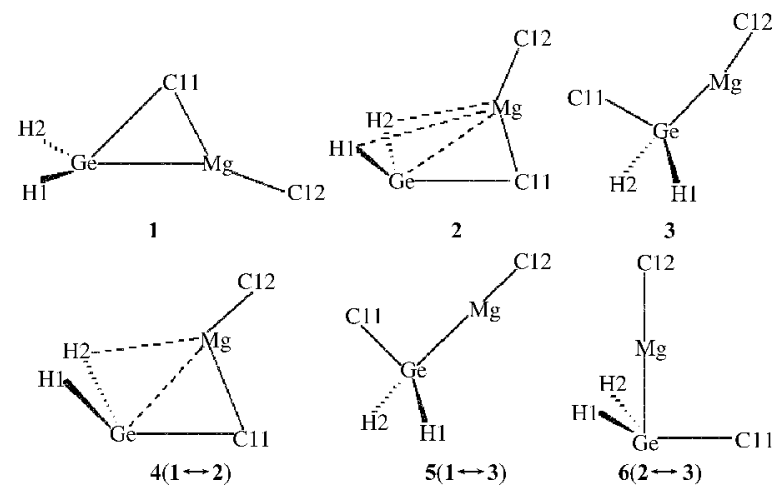

图 $1 \mathrm{H}_{2} \mathrm{GeClMgCl}$ 的构型

Fig.1 The configurations of $\mathrm{H}_{2} \mathrm{GeClMgCl}$ 
表 $1 \mathrm{H}_{2} \mathrm{GeClMgCl}$ 各构型的结构参数

Table 1 The geometric parameters of configurations of $\mathrm{H}_{2} \mathbf{G e C l M g C l}$

\begin{tabular}{|c|c|c|c|c|c|c|c|c|c|}
\hline Structure & $l(\mathrm{GeMg})$ & $l(\mathrm{GeCl1})$ & $l(\mathrm{MgCl})$ & $l(\mathrm{MgCl} 2)$ & $l(\mathrm{GeHl})$ & $A(\mathrm{CllGeMg})$ & $A(\mathrm{Cl} 1 \mathrm{MgCl} 2)$ & $A(\mathrm{H} 1 \mathrm{GeH} 2)$ & $A(\mathrm{Cl} 2 \mathrm{MgGe})$ \\
\hline \multicolumn{10}{|l|}{ gas } \\
\hline 1 & 0.2588 & 0.2509 & 0.2390 & 0.2201 & 0.1553 & 55.9 & 142.1 & 101.9 & 157.5 \\
\hline 2 & 0.2721 & 0.2507 & 0.2380 & 0.2203 & 0.1658 & 54.0 & 142.1 & 85.1 & 159.5 \\
\hline 3 & 0.2575 & 0.2242 & & 0.2202 & 0.1543 & 101.8 & & 106.6 & 179.5 \\
\hline 4 & 0.2665 & 0.2274 & 0.2780 & 0.2216 & 0.1513 & 68.0 & 126.2 & 133.8 & 175.6 \\
\hline 5 & 0.2539 & 0.2290 & & 0.2201 & 0.1543 & 83.7 & & 106.4 & 179.7 \\
\hline 6 & 0.2743 & 0.2216 & & 0.2220 & 0.1521 & 88.8 & & 126.3 & 178.0 \\
\hline \multicolumn{10}{|c|}{ benzene $(\varepsilon=2.247)$} \\
\hline 1 & 0.2581 & 0.2517 & 0.2389 & 0.2208 & 0.1551 & 55.9 & 139.4 & 102.5 & 159.9 \\
\hline 2 & 0.2713 & 0.2526 & 0.2374 & 0.2216 & 0.1668 & 53.8 & 137.8 & 84.4 & 163.1 \\
\hline 3 & 0.2574 & 0.2247 & & 0.2203 & 0.1542 & 100.7 & & 106.8 & 176.6 \\
\hline 4 & 0.2665 & 0.2280 & 0.2762 & 0.2226 & 0.1513 & 67.4 & 123.4 & 134.3 & 173.0 \\
\hline 5 & 0.2540 & 0.2297 & & 0.2205 & 0.1541 & 83.5 & & 107.0 & 174.8 \\
\hline 6 & 0.2738 & 0.2224 & & 0.2229 & 0.1521 & 86.5 & & 127.0 & 172.6 \\
\hline \multicolumn{10}{|c|}{ diethylether $(\varepsilon=4.335)$} \\
\hline 1 & 0.2578 & 0.2526 & 0.2387 & 0.2212 & 0.1550 & 55.8 & 137.6 & 102.9 & 161.4 \\
\hline 2 & 0.2709 & 0.2544 & 0.2370 & 0.2229 & 0.1675 & 53.5 & 134.0 & 84.0 & 166.3 \\
\hline 3 & 0.2573 & 0.2252 & & 0.2204 & 0.1542 & 99.8 & & 107.0 & 173.9 \\
\hline 4 & 0.2666 & 0.2284 & 0.2749 & 0.2233 & 0.1512 & 66.9 & 121.5 & 134.7 & 171.4 \\
\hline 5 & 0.2541 & 0.2304 & & 0.2210 & 0.1540 & 83.5 & & 107.4 & 170.2 \\
\hline 6 & 0.2734 & 0.2230 & & 0.2236 & 0.1520 & 84.4 & & 127.8 & 168.6 \\
\hline \multicolumn{10}{|l|}{ THF $(\varepsilon=7.58)$} \\
\hline 1 & 0.2575 & 0.2531 & 0.2386 & 0.2215 & 0.1549 & 55.7 & 136.5 & 103.1 & 162.4 \\
\hline 2 & 0.2707 & 0.2560 & 0.2368 & 0.2238 & 0.1680 & 53.3 & 131.6 & 83.7 & 168.3 \\
\hline 3 & 0.2572 & 0.2256 & & 0.2205 & 0.1541 & 99.1 & & 107.1 & 172.3 \\
\hline 4 & 0.2666 & 0.2287 & 0.2744 & 0.2237 & 0.1512 & 66.8 & 120.8 & 134.9 & 170.8 \\
\hline 5 & 0.2541 & 0.2311 & & 0.2213 & 0.1539 & 83.2 & & 107.7 & 167.3 \\
\hline 6 & 0.2730 & 0.2235 & & 0.2241 & 0.1520 & 83.0 & & 128.3 & 166.3 \\
\hline \multicolumn{10}{|l|}{ acetone $(\varepsilon=20.7)$} \\
\hline 1 & 0.2573 & 0.2537 & 0.2386 & 0.2218 & 0.1549 & 55.7 & 135.3 & 103.3 & 163.3 \\
\hline 2 & 0.2707 & 0.2582 & 0.2364 & 0.2250 & 0.1686 & 53.0 & 128.6 & 83.4 & 170.6 \\
\hline 3 & 0.2572 & 0.2259 & & 0.2206 & 0.1541 & 98.7 & & 107.2 & 170.3 \\
\hline 4 & 0.2668 & 0.2289 & 0.2741 & 0.2243 & 0.1512 & 66.6 & 119.3 & 135.1 & 169.4 \\
\hline 5 & 0.2541 & 0.2319 & & 0.2217 & 0.1538 & 82.9 & & 107.9 & 164.5 \\
\hline 6 & 0.2727 & 0.2240 & & 0.2247 & 0.1519 & 81.6 & & 128.9 & 164.2 \\
\hline \multicolumn{10}{|l|}{ DMSO $(\varepsilon=46.7)$} \\
\hline 1 & 0.2573 & 0.2539 & 0.2385 & 0.2220 & 0.1549 & 55.6 & 134.8 & 103.4 & 163.7 \\
\hline 2 & 0.2707 & 0.2592 & 0.2362 & 0.2256 & 0.1688 & 52.9 & 127.5 & 83.3 & 171.4 \\
\hline 3 & 0.2571 & 0.2261 & & 0.2206 & 0.1541 & 98.3 & & 107.2 & 169.8 \\
\hline 4 & 0.2669 & 0.2291 & 0.2736 & 0.2244 & 0.1512 & 66.4 & 119.1 & 135.2 & 169.2 \\
\hline 5 & 0.2541 & 0.2323 & & 0.2218 & 0.1538 & 82.7 & & 108.0 & 163.5 \\
\hline 6 & 0.2726 & 0.2242 & & 0.2249 & 0.1519 & 81.2 & & 129.1 & 163.6 \\
\hline
\end{tabular}

$\varepsilon$ : dielectric constant; bond length $(l)$ in $\mathrm{nm}$ and bond angle $(A)$ in degree

看出, 气相下构型 $\mathbf{1}$ 异构为 $\mathbf{2}$ 的活化势垒为 $178.74 \mathrm{~kJ} \cdot \mathrm{mol}^{-1}$, 而构型 $\mathbf{2}$ 异构为 $\mathbf{1}$ 的活化势垒为 $202.84 \mathrm{~kJ} \cdot \mathrm{mol}^{-1}$, 构型 $\mathbf{2}$ 与构型 $\mathbf{1}$ 之间异构化反应 的势垒都较大, 异构化反应较难进行. 构型 3 异构 为 $\mathbf{1}$ 的活化势垒为 $3.01 \mathrm{~kJ} \cdot \mathrm{mol}^{-1}$, 构型 $\mathbf{1}$ 异构为 $\mathbf{3}$ 的活化势垒为 $25.94 \mathrm{~kJ} \cdot \mathrm{mol}^{-1}$, 因此构型 3 易于异 构为构型 $\mathbf{1}$. 构型 $\mathbf{3}$ 异构为 $\mathbf{2}$ 的活化势垒为 159.66 $\mathrm{kJ} \cdot \mathrm{mol}^{-1}$, 构型 $\mathbf{2}$ 异构为 $\mathbf{3}$ 的活化势垒为 206.69 $\mathrm{kJ} \cdot \mathrm{mol}^{-1}$, 二者间的异构化反应也较难进行, 实际上 构型 3 更倾向于异构为构型 $\mathbf{1}$. 通过上述分析可 知, 构型 2 应该是 $\mathrm{H}_{2} \mathrm{GeClMgCl}$ 存在的主要结构, 构型 $\mathbf{1}$ 也有可能存在, 构型 $\mathbf{3}$ 一旦产生则很容易异
构为构型 1 .

\section{3 溶剂效应的影响}

在气相下计算得到的各个平衡构型与过渡态

表 $2 \mathrm{H}_{2} \mathrm{GeClMgCl}$ 各构型的相对能量

Table 2 The relative energies of the geometries for $\mathrm{H}_{2} \mathrm{GeClMgCl}$

\begin{tabular}{crrrrrr}
\hline Structure & \multicolumn{1}{c}{ Gas } & \multicolumn{1}{c}{ Benzene Diethylether } & THF & Acetone & DMSO \\
\hline $\mathbf{1}$ & 24.10 & 27.02 & 29.54 & 31.28 & 33.41 & 34.28 \\
$\mathbf{2}$ & 0.00 & 0.00 & 0.00 & 0.00 & 0.00 & 0.00 \\
$\mathbf{3}$ & 47.03 & 50.79 & 53.73 & 55.75 & 58.08 & 59.04 \\
$\mathbf{4}$ & 202.84 & 204.60 & 206.27 & 207.63 & 209.25 & 209.90 \\
$\mathbf{5}$ & 50.04 & 52.88 & 55.18 & 56.71 & 58.72 & 59.48 \\
$\mathbf{6}$ & 206.69 & 209.03 & 210.75 & 211.86 & 213.26 & 213.81 \\
\hline \multicolumn{7}{c}{ energy in $\mathrm{kJ} \cdot \mathrm{mol}^{-1}$}
\end{tabular}


表 $3 \mathrm{H}_{2} \mathrm{GeClMgCl}$ 的各构型在 DMSO 与气相中的结构参数变化

Table 3 Difference of the geometric parameters for configurations of $\mathrm{H}_{2} \mathrm{GeClMgCl}$ in DMSO and gas phase

\begin{tabular}{crcccccccc}
\hline Structure & $l(\mathrm{GeMg})$ & $l(\mathrm{GeCl})$ & $l(\mathrm{MgCll})$ & $l(\mathrm{MgCl} 2)$ & $l(\mathrm{GeHl})$ & $A(\mathrm{CllGeMg})$ & $A(\mathrm{Cl} 1 \mathrm{MgCl} 2)$ & $A(\mathrm{HlGeH} 2)$ & $A(\mathrm{Cl} 2 \mathrm{MgGe})$ \\
\hline $\mathbf{1}$ & -0.0015 & 0.0030 & -0.0005 & 0.0019 & -0.0004 & -0.3 & -7.3 & 1.5 & 6.2 \\
$\mathbf{2}$ & -0.0014 & 0.0085 & -0.0018 & 0.0053 & 0.0030 & -1.1 & -14.6 & -1.8 & 11.9 \\
$\mathbf{3}$ & -0.0004 & 0.0019 & & 0.0004 & -0.0002 & -3.5 & & 0.6 & -9.7 \\
$\mathbf{4}$ & 0.0004 & 0.0017 & -0.0044 & 0.0028 & -0.0001 & -1.6 & -7.1 & 1.4 & -6.4 \\
$\mathbf{5}$ & 0.0002 & 0.0033 & & 0.0017 & -0.0005 & -1.0 & & 1.6 & -16.2 \\
$\mathbf{6}$ & -0.0017 & 0.0026 & & 0.0029 & -0.0002 & -7.6 & & 2.8 & -14.4 \\
\hline
\end{tabular}

bond length in $\mathrm{nm}$ and bond angle in degree; the difference values are obtained from geometric parameters in DMSO minus those in gas phase.

构型在溶剂中计算依然能够得到, 但构型参数都有 变化, 说明溶剂效应对结构有影响. 从表 1 可以看 出, 随着溶剂的介电常数的增大(即溶剂极性的增大), 构型参数均体现出有规律的变化: 或者随着介电常 数的增大而单调增大, 或者随着介电常数的增大而 单调减小. 表 3 给出了在 DMSO 和气相中结构参 数的变化值. 由于在五种溶剂中 DMSO 的介电常 数最大, 其它溶剂中的构型参数变化值不会超出表 3 所示数值. 从表 3 可看出, 键长变化值并不大, 最 大变化值为构型 $\mathbf{2}$ 中的 $\mathrm{Ge}-\mathrm{Cll}$ 键( 在DMSO 中较 气相下增大 $0.0085 \mathrm{~nm}$ ), 说明溶剂效应对键长大小 影响不大; 但键角变化值较大, 有的键角变化甚至 超过 $10^{\circ}$, 说明溶剂效应对键角的大小影响较大.

从表 2 中可以看出, 在溶液中各构型的能量顺 序与气相中相同, 即溶剂效应不改变各个构型的能 量大小顺序. 随着溶剂的介电常数的增大, 其它构 型对构型 2 的相对能量均增大. 根据相对能量大
小, 可以得出: 随着溶剂的介电常数的增大, 构型 2 异构为 1 和 $\mathbf{3}$ 的势垒均增大, 虽然构型 $\mathbf{1}$ 与 $\mathbf{3}$ 异 构为 2 的势垒有所降低, 但势垒依旧较高, 构型 3 与 $\mathbf{2}$ 及构型 $\mathbf{1}$ 与 $\mathbf{2}$ 之间的异构化反应依然较难进 行; 构型 1 异构为 3 的势垒变化不大, 而构型 3 异 构为 $\mathbf{1}$ 的势垒有明显的降低, 说明随着溶剂极性的 增大, 构型 3 更易于异构化为构型 $\mathbf{1}$. 以在 THF 中 为例, 构型 $\mathbf{2}$ 异构为 $\mathbf{1}$ 和 $\mathbf{3}$ 的势垒分别为 207.63 和 $211.86 \mathrm{~kJ} \cdot \mathrm{mol}^{-1}$, 均比气相下的大(见表 2 ), 构型 1 与 3 异构为 2 的势垒分别为 176.35 和 156.11 $\mathrm{kJ} \cdot \mathrm{mol}^{-1}$, 比气相下稍低(见前), 但势垒依旧较高, 构 型 $\mathbf{3}$ 与 $\mathbf{2}$ 及构型 $\mathbf{1}$ 与 $\mathbf{2}$ 之间的异构化反应较难进 行; 构型 1 异构为 3 的势垒为 $25.43 \mathrm{~kJ} \cdot \mathrm{mol}^{-1}$, 比气 相下的势垒 $\left(25.94 \mathrm{~kJ} \cdot \mathrm{mol}^{-1}\right)$ 稍低, 构型 $\mathbf{3}$ 异构为 $\mathbf{1}$ 的势垒为 $0.96 \mathrm{~kJ} \cdot \mathrm{mol}^{-1}$, 明显低于气相下的势垒 $\left(3.01 \mathrm{~kJ} \cdot \mathrm{mol}^{-1}\right)$, 说明构型 $\mathbf{3}$ 异构为 $\mathbf{1}$ 的反应在 THF 中比气相下容易. 在溶液中各个构型的热力学
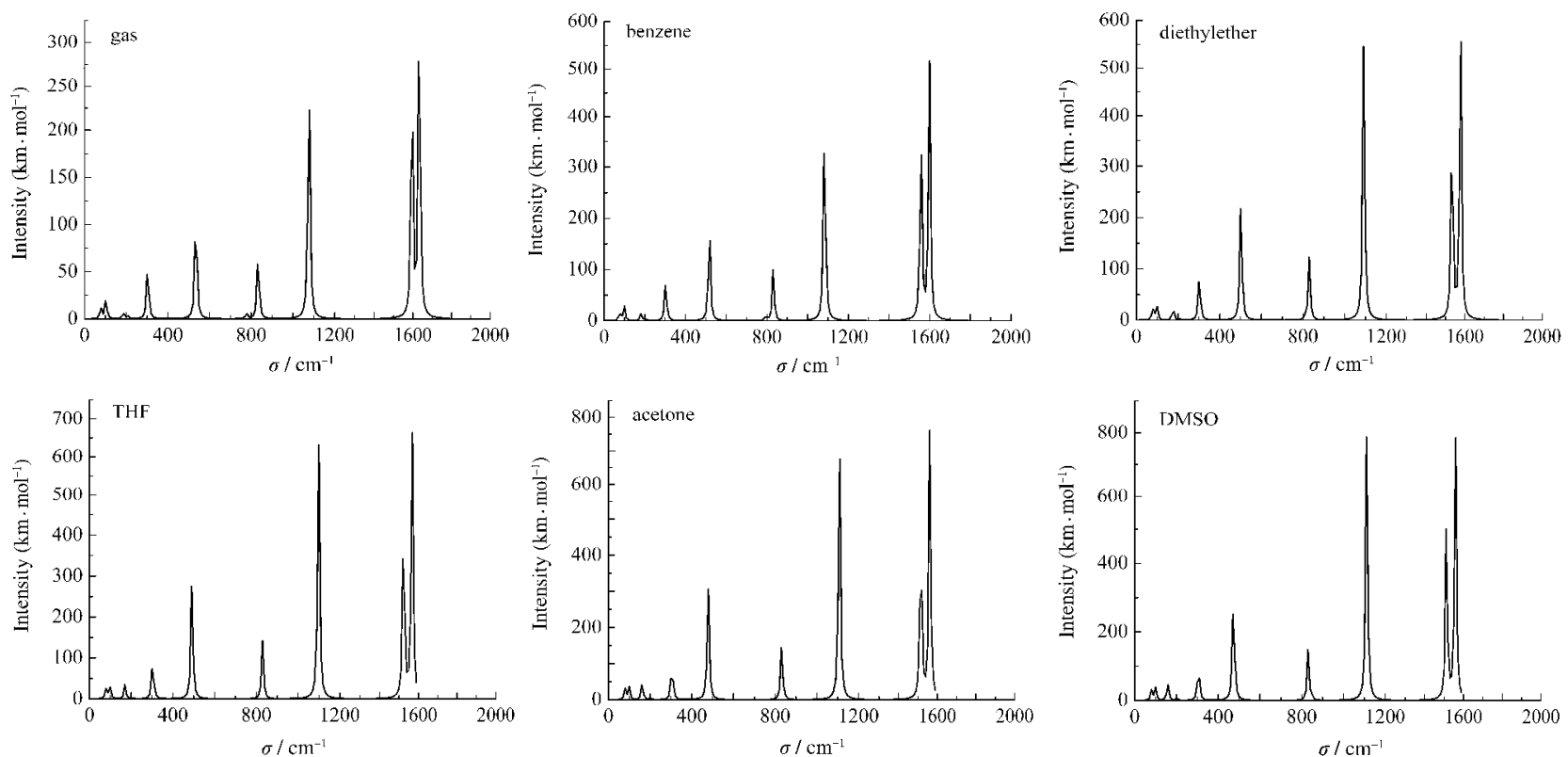

图 2 在气相和五种溶剂中模拟的 $\mathrm{H}_{2} \mathrm{GeClMgCl}$ 的构型 2 的红外光谱

Fig.2 The simulated infrared spectra of structure 2 of $\mathrm{H}_{2} \mathrm{GeClMgCl}$ in gas phase and five solvents 
稳定性与动力学稳定性与气相下相同.

由上所述可知, 不论在气相还是在溶剂中, 构 型 2 均是最稳定的构型. 图 2 给出了在气相和五种 溶剂中根据 B3LYP/6-311+G $(d, p)$ 的频率计算结果 模拟的构型 $\mathbf{2}$ 的红外光谱图, 可以为实验提供理论 参考. 从图中可以看出, 波数为 $1600 \mathrm{~cm}^{-1}$ 附近的双 峰 (对应 $\mathrm{Ge}-\mathrm{H}$ 伸缩振动) 随溶剂的介电常数增大 而红移, 这与 $\mathrm{Ge}-\mathrm{H}$ 键随溶剂的介电常数增大而 变长(见表 1 )相符合.

\section{3 结 论}

应用 DFT(B3LYP) 与 QCISD 方法研究了类锗 烯 $\mathrm{H}_{2} \mathrm{GeClMgCl}$ 在气相及五种溶剂苯、乙醚、四氢 呋喃、丙酮和二甲基亚砜中的结构及异构化反应. 计 算结果表明, 类锗烯 $\mathrm{H}_{2} \mathrm{GeClMgCl}$ 有 3 种可能的平 衡构型: 三元环构型、 $p$-配合物型构型和 “四面体”构 型, 其中 $p$-配合物型构型能量最低, 是其存在的主 要构型. 通过比较各个构型在气相和五种溶剂中的 结构参数与能量, 发现溶剂效应对各异构体的键长 影响不大, 对键角影响较大. 在溶液中各个构型的 热力学稳定性与动力学稳定性与气相下相同. 计算 模拟了最稳定构型的红外光谱. 本文内容为进一步 研究类锗烯提供了有用信息.

\section{References}

1 Lei, D. Q.; Gaspar, P. P. Polyhedron, 1991, 10: 1221

2 Sekiguchi, A.; Lee, V. Y. Chem. Rev., 2003, 103: 1429

3 Tajima, A.; Sasamori, T.; Takeda, N.; Tokitoh, N.; Yoshida, K.; Nakahara, M. Organometallics, 2006, 25: 230

4 Sasamori, T.; Tokitoh, N. Organometallics, 2006, 25: 3522

5 Satge, J. Pure Appl. Chem., 1984, 56: 137

6 Tokitoh, N.; Kishikawa, K.; Okazaki, R. Polyhedron, 2002, 21: 563

7 Iwamoto, T.; Masuda, H.; Ishida, S. J. Organomet. Chem., 2004, 689: 1337

8 Kassaee, M. Z.; Ghambarian, M.; Musavi, S. M. J. Organomet. Chem., 2005, 690: 4692
9 Qiu, H. Y.; Ma, W. Y.; Li, G. B.; Deng, C. H. Chin. Chem. Lett., 1999, 10: 511

10 Tan, X.; Li, P.; Wang, D.; Yang, X. J. Mol. Struct. -Theochem, 2006, 761: 27

11 Tan, X.; Li, P.; Yang, X.; Wang, D. Int. J. Quantum Chem., 2006, 106: 1902

12 Zhu, Y. F.; Fang, Y. Z.; Zhou, J. H.; Ma, W. Y. Chin. J. Struct. Chem., 2007, 26: 395

13 Ma, W. Y.; Zhu, Y. F.; Zhou, J. H.; Fang, Y. Z. J. Mol. Struct. Theochem, 2007, 817: 77

14 Li, W. Z.; Cheng, J. B.; Gong, B. A.; Xiao, C. P. J. Organomet. Chem., 2006, 691: 5984

15 Li, W. Z.; Tan, H. N.; Xiao, C. P.; Gong, B. A.; Cheng, J. B. Acta Phys. -Chim. Sin., 2007, 23: 1811 [李文佐, 谭海娜, 肖翠 平, 宫宝安, 程建波. 物理化学学报, 2007, 23: 1811]

16 Li, W. Z.; Xiao, C. P.; Gong, B. A.; Cheng, J. B. Acta Phys.Chim. Sin., 2008, 24: 720 [李文佐, 肖翠平, 宫宝安, 程建波. 物理化学学报, 2008, 24: 720]

17 Li, W. Z.; Gong, B. A.; Cheng, J. B.; Xiao, C. P. J. Mol. Struct. Theochem, 2007, 847: 75

18 Hohenberg, P.; Kohn, W. Phys. Rev., 1964, 136: B864

19 Feller, D. J. Chem. Phys., 1990, 93: 579

20 Becke, A. D. J. Chem. Phys., 1993, 98: 5648

21 Lee, C.; Yang, W.; Parr, R. G. Phys. Rev., 1988, 37: 785

22 Gonzalez, C.; Schlegel, H. B. J. Chem. Phys., 1989, 90: 2154

23 Gonzalez, C.; Schlegel, H. B. J. Phys. Chem., 1990, 94: 5523

24 Hehre, W. J.; Radom, L.; Schleyer, P. von R.; Pople, J. A. Ab initio molecular orbital theory. New York: Wiley, 1986

25 Gauss, J.; Cremer, C. Chem. Phys. Lett., 1988, 150: 280

26 Salter, E. A.; Trucks, G. W.; Bartlett, R. J. J. Chem. Phys., 1989, 90: 1752

27 Li, W. Z.; Cheng, J. B.; Gong, B. A.; Yu, J. K.; Sun, J. Z. Acta Phys. -Chim. Sin., 2008, 24: 901 [李文佐, 程建波, 宫宝安, 于 健康, 孙家钟. 物理化学学报, 2008, 24: 901]

28 Wong, M. W.; Frisch, M. J.; Wiberg, K. B. J. Am. Chem. Soc., 1991, 113: 4776

29 Ai, H. Q.; Yang, A. B.; Li, Y. G. Acta Phys.-Chim. Sin., 2008, 24: 1047 [艾洪奇, 杨爱涁, 李允刚. 物理化学学报, 2008, 24: 1047]

30 Frisch, M. J.; Trucks, G. W.; Schlegel, H. B.; et al. Gaussian 03, Revision B.03. Pittsburgh, PA: Gaussian Inc., 2003 